\title{
Clinical Study \\ Evaluation of Hemodynamic Parameters as Predictors of Glaucoma Progression
}

\author{
Ingrida Janulevičiene, ${ }^{1}$ Rita Ehrlich, ${ }^{2}$ Brent Siesky, ${ }^{2}$ Irena Nedzelskienè, ${ }^{3}$ and Alon Harris ${ }^{2}$ \\ ${ }^{1}$ Eye Clinic, Kaunas University of Medicine, Eiveniu Street 2, 50009 Kaunas, Lithuania \\ ${ }^{2}$ Department of Ophthalmology, Glaucoma Research and Diagnostic Center, Indiana University School of Medicine, \\ 702 Rotary Circle, Room 137, Indianapolis, IN 46202, USA \\ ${ }^{3}$ Biostatistician, Faculty of Odontology, Kaunas University of Medicine, 50106 Kaunas, Lithuania \\ Correspondence should be addressed to Ingrida Janulevičiene, ingrida.januleviciene@kmuk.lt \\ Received 3 June 2010; Revised 17 August 2010; Accepted 15 February 2011 \\ Academic Editor: Christopher Kai-shun Leung
}

Copyright (@ 2011 Ingrida Janulevičiene et al. This is an open access article distributed under the Creative Commons Attribution License, which permits unrestricted use, distribution, and reproduction in any medium, provided the original work is properly cited.

Purpose. To evaluate hemodynamic parameters as possible predictors for glaucoma progression. Methods. An 18-month randomized double-masked cohort study including 30 open-angle glaucoma patients receiving fixed-combination treatment with Dorzolamide/Timolol (DTFC) or Latanoprost/Timolol (LTFC) $(n=15$ per group) was performed. Intraocular pressure (IOP), arterial blood pressure (BP), ocular and diastolic perfusion pressures (OPP, DPP), color Doppler imaging, pulsatile ocular blood flow analysis, scanning laser polarimetry, and Humphrey visual field evaluations were included. Results. Both treatments showed statistically similar IOP reduction. Six patients in DTFC and 7 in LTFC group met glaucoma progression criteria. DTFC group had higher OPP, DPP, and lower vascular resistivity indices as compared to the LTFC. Progressing patients had higher nerve fiber index, lower systolic BP, OPP, DPP, higher ophthalmic and central retinal artery vascular resistance, and lower pulse volume $(P<.05$; $t$-test). Conclusions. Structural changes consistent with glaucoma progression correlate with non-IOP-dependent risk factors.

\section{Introduction}

The recent series of large, multicenter, randomized clinical trials examining glaucoma treatment provide some information regarding current management goals for maintaining a target intraocular pressure (IOP). However, in many cases, glaucoma progression occurs despite maintaining target IOP. For instance, in the Collaborative Normal-Tension Glaucoma (CNTG) study, 12 to $18 \%$ of glaucoma patients progressed despite a 30\% IOP reduction [1]; in the Early Manifest Glaucoma Trial (EMGT), 45\% progressed despite an average IOP reduction of $25 \%$ at 6 -year followup [2]. Leske et al. [3] further reported that $67 \%$ of patients progressed over 11 years of followup despite IOP reduction.

Non-IOP factors have also been identified as contributing to open-angle glaucoma (OAG) progression, including lower ocular perfusion pressure (OPP), reduced ocular blood flow, cardiovascular disease, and low systolic blood pressure. Impaired optic nerve blood flow is considered a potential causative factor in the development of glaucoma optic neuropathy $[4,5]$. However, it remains unknown whether manipulation of perfusion pressure, blood pressure, and ocular blood flow will prevent glaucoma progression.

The European Glaucoma Guidelines of 2008 [6] set the preservation of visual function as the primary goal of glaucoma therapy. In cellular terms, this can be interpreted as prevention of retinal ganglion cell death. However, the exact factors contributing to retinal ganglion cell death remain speculative [7]. Although changes in ocular blood flow might be the consequence of IOP variations, they can also be a primary physiological event [8]. As IOP therapies may influence ocular perfusion [9], it is vital to investigate glaucoma therapies for vascular interactions in addition to IOP reduction. One possible therapy is dorzolamide hydrochloride, a potent vasoactive glaucoma topical treatment that many studies have shown to increase various measures of ocular blood flow [10-16]. Although not all studies are in full agreement $[17,18]$, a recent 
meta-analysis of published studies found carbonic anhydrase inhibitors, such as dorzolamide, to be consistently effective at increasing the ocular circulation [19].

Much less research has been conducted to investigate the effects of a combination treatment on improving ocular circulation and reducing IOP, especially in relation to glaucoma progression. To our knowledge, there are no long-term prospective double-blind studies that investigated the IOP lowering effects of fixed combinations and the correlation between ocular hemodynamic and both functional and structural changes in glaucoma patients. This study investigates the fixed combinations of dorzolamide/timolol (DTFC) and latanoprost/timolol (LTFC) on IOP lowering and glaucoma progression while examining if baseline ocular blood flow parameters are predictive of glaucomatous progression as determined by visual field and/or structural changes.

\section{Materials and Methods}

Thirty OAG patients were followed for 18 months in an observational cohort study. All subjects read and signed an informed consent, and the study was approved by the Kaunas University of Medicine institutional review board. Inclusion criteria: OAG patients with characteristic glaucomatous visual field loss, optic nerve head damage, and IOP not adequately controlled with timolol maleate (BID). Exclusion criteria: mean deviation worse than or equal to $-12 \mathrm{~dB}$ in Humphrey Visual Fields (HVFA) central 24-2 SITA Standard, cup to disc ratio equal or greater than 0.9 , history of eye disease other than refractive error, orbital or ocular trauma, history of renal or hepatic disease, asthma or respiratory disease, allergy to either of the study medications, and pregnant or nursing women. After timolol baseline examination, patients were randomly assigned to double masked fixed combination treatment: LTFC or DTFC. Examinations were carried out in both eyes and the study eye was chosen randomly. All study visits were scheduled at the same time of day \pm 1 hour in order to avoid diurnal fluctuations in IOP and arterial BP.

Examinations were carried out at baseline, 1, 6, 12, and 18 months of treatment, including full ophthalmic examination, visual acuity, Goldmann IOP, central corneal thickness (CCT) (OcuScan PXP Alcon Labs. Inc), Humphrey visual field examination (24-2 SITA Standard), and scanning laser polarimetry (GDx VCC Laser Diagnostic Technologies Inc., San Diego, CA). In the scanning laser polarimetry scan printout each color represents a different probability of the parameter being outside normal limits, with red having the highest probability $(P<.005)$, followed by yellow $(P<.01)$, light blue $(P<.02)$, and dark blue $(P<.05)$; green $(P<.05)$ refers to normal limits.

All patients had 5 or more visual fields and scanning laser polarimetry scans for analysis. Glaucoma progression was identified by (1) standard automated perimetry (SAP) as a statistically significant decrease from baseline examination in the pattern deviation values. Deepening of an existing scotoma was considered if two points in an existing scotoma declined by $\geq 10 \mathrm{~dB}$. Expansion of an existing scotoma was considered if two contiguous points adjacent to an existing scotoma declined by $\geq 10 \mathrm{~dB}$. A new scotoma was diagnosed if an alteration meeting the criteria for glaucomatous visual field defect occured in previously normal visual field location. Three or more locations with $P<.01$ constituted a change of threshold sensitivity. (2) Progressive optic disc change is determined by optic disc assessment by ophthalmoscopy and scanning laser polarimetry. Advanced Serial Analysis detected repeatable change on two consecutive scans compared with baseline images using thickness map, and deviation map, deviation from reference map, temporal-superior-nasal-inferior-temporal (TSNIT) graph or a significant change in slope of the summary parameter chart. Each slope represented the change in RNFL thickness per year, assuming a linear trend across the followup period [3, 20-22].

Ocular blood flow was evaluated with pulsatile ocular blood flow analyser POBF (Paradigm medical industries. Inc.) and Color Doppler imaging (CDI) (Accuvix XQ. Medison Co., LTD. Seoul, Republic of Korea). Blood flow velocities were measured in the ophthalmic (OA), central retinal (CRA), and short posterior ciliary arteries (SPCA), with a $7.5 \mathrm{MHz}$ linear probe calculating peak systolic velocity (PSV), end-diastolic velocity (EDV), and resistive index (RI) in each vessel. Vascular RI was originally described by Pourcelot and is calculated as RI $=(\mathrm{PSV}-\mathrm{EDV}) / \mathrm{PSV}$ [23-26].

All patients' data were collected in the Eye Clinic of Kaunas Medical University (Lithuania). CDI readings were performed by a Reading Center: the Glaucoma Research and Diagnostic Laboratories in the Department of Ophthalmology, Indiana University School of Medicine (USA).

\section{Statistical Analysis}

CDI presents 12 different parameters with a coefficient of variation ranging from $1.7 \%$ to $18 \%$, and the majority of parameters present with a coefficient of variation under $10 \%$. The coefficient of variation for total RNFL thickness is $5 \%$. With a sample size of 15 in each group, we have at least $90 \%$ power to detect a change as small as $8.5 \%$ with alpha level 0.05 in retrobulbar velocities and $4.2 \%$ in RNFL thickness. The coefficient of variation for POBF is $15 \%$ [24]. In this analysis, we determined our sample size must be greater than 29.17 subjects to detect changes smaller than $9 \%$ in blood flow parameters. Changes in visual fields over time were analyzed using Humphrey's STATPAC software as described in Materials and Methods.

Descriptive statistics were obtained for the resulting measurements. In the event that significance was achieved by repeated ANOVA measurements, we applied the Fisher's and Bonferroni models. Changes in individual parameters were examined by paired Student's $t$-test. $P$ values of $P<.05$ were considered statistically significant. To test the hypothesis that the mean difference between two measurements is zero, Wilcoxon signed-ranks test was used. Changes in OBF and glaucomatous optic neuropathy parameters (functional and structural changes) were analyzed by Pearson's correlation analysis. Multivariate regression models were used to evaluate potential risk factors for glaucoma progression: age, IOP, systolic BP, diastolic BP, OPP, DPP, pulse volume, and RI of 


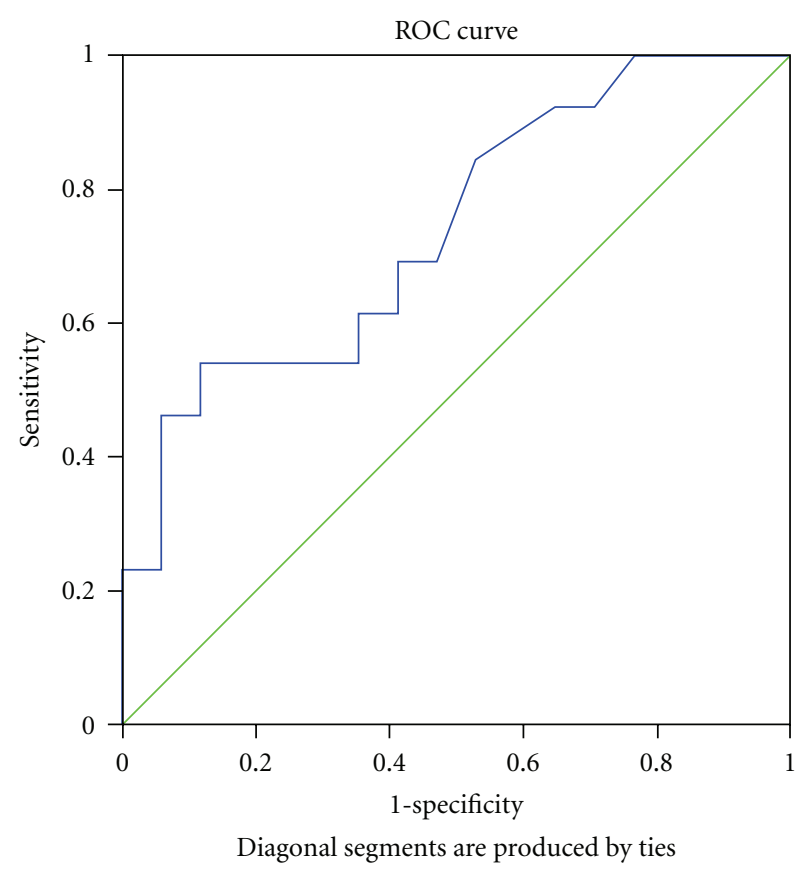

Figure 1: ROC curve-DPP at 18-month visit in progressing glaucoma patients. ROC: Receiver operating characteristic.

retrobulbar vessels. Receiver Operating Characteristic (ROC) curves for progressing glaucoma patients were performed to analyze the discriminating ability of possible vascular risk factors.

\section{Results}

We examined 30 OAG patients (15 patients in each study group) with a mean age of 58.13 (SD 8.6), including 5 males and 25 females. There were no statistically significant differences between baseline parameters of either treatment group.

Both DTFC and LTFC had similar IOP lowering effect over 18 months of observation $(P=.653$; $t$-test $)$. Baseline systolic and diastolic BP were comparable between DTFC and LTFC groups $(P=0.101$ and $P=0.07$, resp., $t$-test $)$. DTFC showed statistically significantly higher OPP, SPP, and DPP at 1, 6, and 18 months visits (Table 1 ).

CDI baseline retrobulbar blood flow parameters were similar between the two groups $(P>.05 ; t$-test $)$, except for a statistically significantly higher OA-PSV and CRA-EDV in the LTFC group (Table 2). Both combination treatment regimes increased retrobulbar blood flow velocities compared to baseline, though significant changes from baseline at the OA-PSV $(P=.003)$, OA-EDV $(P=.001)$, and CRA-PSV $(P=.001)$ were only seen in the DTFC group at 1- and 12-month followup. Vascular RI were decreased in the DTFC group, showing statistically significantly lower resistivity compared to the LTFC group in the CRA and SPCA during 12- and 18-month visits (Table 2). CRA-PSV correlated with OA-PSV $(r=0.505 ; P=.004)$ and OA-EDV $(r=0.450 ; P=.013)$, and SPCA-EDV correlated with DBP

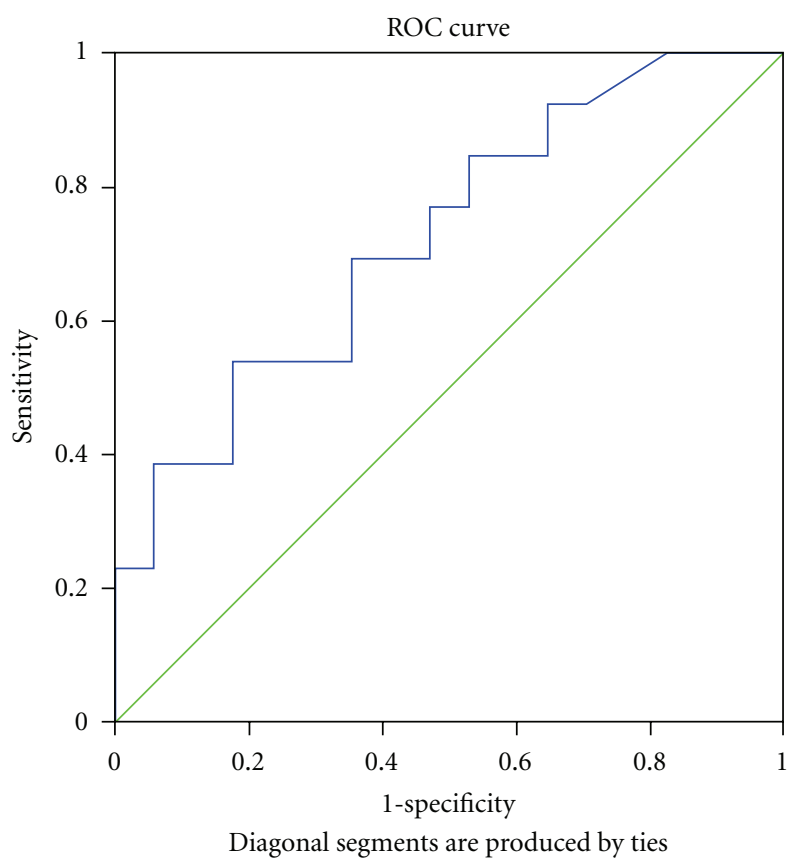

FIGURE 2: ROC curve-OPP at 18-month visit in progressing glaucoma patients. ROC: Receiver operating characteristic.

$(r=0.454 ; P=.012)$, DPP $(r=0.449 ; P=.013)$, and OA-RI $(r=-0.432 ; P=.017)$.

Average IOP, pulse amplitude, and POBF were not statistically different between treatment arms (Table 3). Pulse volume increases in the DTFC group and differences at the 12- and 18-month visits when compared to the LTFC group were significant $(P=.025$ and $P=.054$, resp. $)$.

Glaucoma progression was identified in 13 eyes $(21.7 \%)$ : 4 (6.7\%) exhibiting structural changes, 1 (1.7\%) with perimetric changes, and $8(13.3 \%)$ showing both perimetric and structural changes. There were no statistically significant differences in IOP between progressing and stable glaucoma patients at the final visit (Table 4). Progressing glaucoma patients had higher OA RI, lower SPCA-EDV $(P<.05 ; t$ test), and decreased pulse volume by 2.68 (SD 0.61) $\mu \mathrm{L}(P=$ $.0001 ; t$-test) as compared to stable glaucoma patients at the 18 -month visit. Progressing glaucoma cases had significantly lower SBP, OPP, and DPP (Table 4).

Changes in TSNIT correlated with SBP $(r=0.614 ; P=$ $.025)$ in progressing glaucoma patients. The odds of higher $\mathrm{NFI}$ at the final 18 -month visit was 13.82 times greater $(95 \%$ CI 1.32-143.76) in patients with baseline CRA RI $\geq 0.67$ $(P=.028)$ and older age patients (95\% CI 0.90-0.99) $(P=$ $.021)$.

The area under the Receiver Operating Characteristic (ROC) curve in progressing glaucoma patients with DPP < $62 \mathrm{mmHg}$ was 0.74 (95\% CI lower bound 0.56; upper bound $0.919 ; P=.027$ ) (Figure 1 ); the sensitivity and specificity were 0.385 and 0.941 , respectively. Progressing glaucoma patients with OPP $<52 \mathrm{mmHg}$ had an area under the ROC curve of 0.72 ( $95 \%$ CI lower bound 0.54; upper bound 0.907; $P=.038$ ) (Figure 2); the sensitivity and specificity were 
TABLE 1: Comparison of characteristics of patients treated with DTFC and LTFC.

\begin{tabular}{|c|c|c|c|}
\hline Characteristics & DTFC & LTFC & $\begin{array}{l}P \text { value } \\
(t \text {-test })\end{array}$ \\
\hline Age & $56.93(9.54)$ & $59.33(7.7)$ & .455 \\
\hline $\operatorname{CCT}(\mu)$ & $548.03(39.86)$ & $549.65(41.71)$ & .914 \\
\hline $\mathrm{C} / \mathrm{D}$ ratio & $0.62(0.14)$ & $0.65(0.15)$ & .576 \\
\hline SBP mmHg baseline & $157.70(14.90)$ & $146.70(20.22)$ & .101 \\
\hline 1 month & $152.73(16.90)$ & $136.00(13.67)$ & $.006^{*}$ \\
\hline 6 months & $161.80(18.40)^{*}$ & $146.800(15.40)^{*}$ & $.022 *$ \\
\hline 12 months & $148.500(11.18)$ & $144.200(17.41)$ & .428 \\
\hline 18 months & $158.63(14.24)$ & $141.10(15.21)$ & $.003^{*}$ \\
\hline DBP mmHg baseline & $92.13(8.12)$ & $86.80(7.53)$ & .073 \\
\hline 1 month & $93.73(15.41)$ & $81.10(7.04)$ & $.009^{*}$ \\
\hline 6 months & $97.43(12.19)^{*}$ & $86.87(9.49)^{*}$ & $.013^{*}$ \\
\hline 12 months & $91.07(8.47)$ & $86.57(9.10)$ & .172 \\
\hline 18 months & $88.80(5.81)$ & $83.83(8.41)$ & .070 \\
\hline IOP mmHg baseline & $22.10(2.69)$ & $20.57(3.25)$ & .171 \\
\hline 1 month & $16.33(2.11)$ & $14.90(2.69)$ & .116 \\
\hline 6 months & $16.17(2.81)$ & $14.70(2.57)$ & .147 \\
\hline 12 months & $17.10(2.42)$ & $15.13(3.42)$ & .080 \\
\hline 18 months & $16.17(2.08)$ & $15.70(3.38)$ & .653 \\
\hline OPP mmHg baseline & $53.8933(5.61)$ & $50.6100(7.52)$ & .186 \\
\hline 1 month & $59.27(9.70)^{*}$ & $51.47(4.6)^{*}$ & $.011^{*}$ \\
\hline 6 months & $62.93(8.98)^{*}$ & $56.33(5.84)^{*}$ & $.024^{*}$ \\
\hline 12 months & $56.38(6.19)$ & $55.38(6.92)$ & .683 \\
\hline 18 months & $57.56(3.81)$ & $52.18(7.26)$ & $.019^{*}$ \\
\hline SPP mmHg baseline & $135.60(7.40)$ & $126.13(10.51)$ & $.008^{*}$ \\
\hline 1 month & $136.40(12.1)$ & $121.10(7.5)$ & $.003^{*}$ \\
\hline 6 months & $145.63(19.6)$ & $132.10(8.4)$ & $.020^{*}$ \\
\hline 12 months & $131.4(8.25)$ & $129.07(10.24)$ & .498 \\
\hline 18 months & $142.46(7.4)$ & $125.40(9.34)$ & $.0001^{*}$ \\
\hline DPP mmHg baseline & $70.03(7.40)$ & $66.23(8.11)$ & .191 \\
\hline 1 month & $77.20(15.12)^{*}$ & $66.73(5.35)^{*}$ & $.021^{*}$ \\
\hline 6 months & $81.33(12.19)^{*}$ & $71.67(7.95)^{*}$ & $.016^{*}$ \\
\hline 12 months & $73.97(8.41)$ & $71.43(8.90)$ & .430 \\
\hline 18 months & 72.97 (6.15) & $66.03(11.03)$ & $.045^{*}$ \\
\hline
\end{tabular}

${ }^{*} P<.05$ statistically significant.

DTFC: dorzolamide/timolol fixed combination; LTFC: latanoprost/timolol fixed combination; CCT: central corneal thickness; C/D ratio: clinically determined cup-disc ratio; SBP: systolic blood pressure; DBP: diastolic blood pressure; IOP: intraocular pressure; OPP: ocular perfusion pressure; DPP: diastolic perfusion pressure.

0.385 and 0.941 , respectively. In our analysis, we found power 0.88 with type I error of 0.05 and, although sensitivity was low at cut off, the specificity was high.

\section{Discussion}

This observational cohort study showed that despite the IOP lowering effect with different fixed combinations (DTFC and LTFC), 13 eyes (21.7\%) were considered as progressing glaucoma during 18 months of observation. Among patients with progressing glaucoma, 6 were with DTFC and 7 with LTFC treatment and showed no statistically significant hypotensive effect between the two fixed combinations. Evidence shows that despite a wide range of glaucoma therapy options to reduce IOP, it is still difficult in some cases to control slowly progressing optic neuropathy. During our 18-month observation, no cases of intolerance were found and all patients completed the study.

Previously, Siesky et al. [27] reported that DTFC increased ocular blood flow in OAG patients while attaining a similar IOP reduction compared to a treatment of latanoprost plus timolol. Visual function, as expected, was not different in this short-term comparison. Evidence of decreased optic nerve blood flow correlating with visual field 
TABLE 2: Color Doppler imaging parameters during 18 months of followup.

\begin{tabular}{|c|c|c|c|c|}
\hline \multicolumn{2}{|c|}{ Characteristics } & \multirow{2}{*}{$\begin{array}{c}\text { DTFC } \\
23.79(8.837)\end{array}$} & \multirow{2}{*}{$\begin{array}{c}\text { LTFC } \\
30.86(9.30)\end{array}$} & \multirow{2}{*}{$\begin{array}{c}\begin{array}{c}P \text { value } \\
(t \text {-test })\end{array} \\
.042\end{array}$} \\
\hline \multirow{5}{*}{ OA_PSV (cm/s) } & Baseline & & & \\
\hline & 1 month & $37.10(12.33)$ & $36.04(7.83)$ & .781 \\
\hline & 6 months & $38.15(16.24)$ & $33.87(8.27)$ & .371 \\
\hline & 12 months & $40.66(15.51)$ & $42.50(14.01)$ & .736 \\
\hline & 18 months & $33.70(10.05)$ & $28.71(6.93)$ & .125 \\
\hline \multirow{5}{*}{ OA_EDV $(\mathrm{cm} / \mathrm{s})$} & Baseline & $4.82(2.47)$ & $7.03(3.60)$ & .06 \\
\hline & 1 month & $8.22(4.22)$ & $8.78(3.94)$ & .710 \\
\hline & 6 months & $8.87(6.03)$ & $7.66(2.52)$ & .479 \\
\hline & 12 months & $10.59(4.79)$ & $9.63(5.11)$ & .599 \\
\hline & 18 months & $9.47(6.19)$ & $7.23(4.54)$ & .268 \\
\hline \multirow{5}{*}{ OA_RI } & Baseline & $0.79(0.11)$ & $0.76(0.11)$ & .437 \\
\hline & 1 month & $0.79(0.07)$ & $0.75(0.11)$ & .158 \\
\hline & 6 months & $0.76(0.11)$ & $0.76(0.09)$ & .986 \\
\hline & 12 months & $0.72(0.12)$ & $0.82(0.17)$ & $.046^{*}$ \\
\hline & 18 months & $0.76(0.10)$ & $0.87(0.28)$ & .189 \\
\hline \multirow{5}{*}{ CRA_PSV $(\mathrm{cm} / \mathrm{s})$} & Baseline & $15.09(3.78)$ & $17.91(7.80)$ & .218 \\
\hline & 1 month & $17.78(4.43)$ & $18.59(7.34)$ & .716 \\
\hline & 6 months & $19.08(7.59)$ & $17.67(5.95)$ & .575 \\
\hline & 12 months & $28.88(13.40)$ & $22.71(12.82)$ & .208 \\
\hline & 18 months & $18.69(8.79)$ & $17.46(5.24)$ & .645 \\
\hline \multirow{5}{*}{ CRA_EDV $(\mathrm{cm} / \mathrm{s})$} & Baseline & $4.56(1.81)$ & $6.33(2.48)$ & $.034^{*}$ \\
\hline & 1 month & $6.49(2.22)$ & $5.41(3.19)$ & .291 \\
\hline & 6 months & $6.0(2.49)$ & $6.16(2.64)$ & .868 \\
\hline & 12 months & $7.56(3.67)$ & $10.31(7.34)$ & .204 \\
\hline & 18 months & $5.66(2.80)$ & $6.85(3.24)$ & .289 \\
\hline \multirow{5}{*}{ CRA_RI } & Baseline & $0.80(0.26)$ & $0.81(0.25)$ & .915 \\
\hline & 1 months & $0.68(0.08)^{*}$ & $0.80(0.16)^{*}$ & $.011^{*}$ \\
\hline & 6 months & $0.65(0.082)$ & $0.72(0.19)$ & .192 \\
\hline & 12 months & $0.74(0.19)$ & $0.85(0.19)$ & $.000^{*}$ \\
\hline & 18 months & $0.67(0.09)$ & $0.93(0.23)$ & $.000^{*}$ \\
\hline \multirow{5}{*}{ SPCA_PSV (cm/s) } & Baseline & $15.55(4.70)$ & $14.50(6.59)$ & .606 \\
\hline & 1 month & $15.95(5.91)$ & $13.38(3.10)$ & .147 \\
\hline & 6 months & $20.03(6.42)$ & $17.92(3.68)$ & .280 \\
\hline & 12 months & $21.01(10.40)$ & $19.81(7.04)$ & .715 \\
\hline & 18 months & $13.69(5.45)$ & $11.03(2.83)$ & .104 \\
\hline \multirow{5}{*}{ SPCA_EDV $(\mathrm{cm} / \mathrm{s})$} & Baseline & $4.42(2.29)$ & $14.50(6.59)$ & .973 \\
\hline & 1 month & $4.69(2.28)$ & $3.31(2.11)$ & .095 \\
\hline & 6 months & $6.10(2.16)$ & $5.47(2.22)$ & .442 \\
\hline & 12 months & $6.04(2.67)^{*}$ & $3.43(2.26)^{*}$ & $.007^{*}$ \\
\hline & 18 months & $4.39(1.85)$ & $3.87(1.17)$ & .366 \\
\hline \multirow{5}{*}{ SPCA_RI } & Baseline & $0.71(0.06)$ & $0.79(0.28)$ & .232 \\
\hline & 1 month & $0.75(0.08)$ & $0.79(0.10)$ & .229 \\
\hline & 6 months & $0.69(0.06)$ & $0.69(0.11)$ & .969 \\
\hline & 12 months & $0.70(0.07)^{*}$ & $0.90(0.27)^{*}$ & $.011^{*}$ \\
\hline & 18 months & $0.69(0.11)^{*}$ & $0.85(0.30)^{*}$ & $.015^{*}$ \\
\hline
\end{tabular}

${ }^{*} P<.05$ statistically significant.

DTFC: dorzolamide/timolol fixed combination; LTFC: latanoprost/timolol fixed combination; OA: ophthalmic artery; CRA: central retinal artery; SPCA: short posterior ciliary artery, PSV: peak systolic velocity; EDV: end diastolic velocity; RI: resistive index. 
TABLe 3: Pulsatile ocular blood flow parameters.

\begin{tabular}{|c|c|c|c|c|}
\hline \multicolumn{2}{|c|}{ Characteristics } & DTFC & LTFC & $P$ value \\
\hline \multirow{5}{*}{ IOP average ( $\mathrm{mmHg})$} & baseline & $19.58(3.68)$ & $20.96(3.78)$ & .320 \\
\hline & 1 month & $17.12(3.25)$ & $18.01(2.83)$ & .429 \\
\hline & 6 months & $17.67(3.73)$ & $17.71(3.17)$ & .975 \\
\hline & 12 months & $17.87(3.59)$ & $16.48(2.56)$ & .231 \\
\hline & 18 months & $16.10(2.78)$ & $15.23(4.61)$ & .539 \\
\hline \multirow{5}{*}{ Pulse amplitude } & Baseline & $4.17(1.50)$ & $4.73(1.58)$ & .335 \\
\hline & 1 month & $3.91(0.88)$ & $3.95(1.18)$ & .917 \\
\hline & 6 months & $4.93(1.88)$ & $4.12(1.47)$ & .201 \\
\hline & 12 months & $4.75(1.40)$ & $4.67(1.74)$ & .891 \\
\hline & 18 months & $4.73(2.78)$ & $4.51(1.42)$ & .675 \\
\hline \multirow{5}{*}{ Pulse volume $(\mu \mathrm{L})$} & Baseline & $7.19(2.36)$ & $7.81(2.68)$ & .507 \\
\hline & 1 month & $7.99(2.27)$ & $7.60(2.40)$ & .648 \\
\hline & 6 months & $8.91(2.23)$ & $7.07(3.26)$ & .417 \\
\hline & 12 months & $9.25(1.95)^{*}$ & $6.93(3.20)^{*}$ & $.025^{*}$ \\
\hline & 18 months & $9.29(2.39)^{*}$ & $7.82(1.55)$ & $.054^{*}$ \\
\hline \multirow{5}{*}{ POBF Baseline $(\mu \mathrm{L} / \mathrm{s})$} & Baseline & $16.81(4.53)$ & $17.57(6.13)$ & .702 \\
\hline & 1 month & $19.12(4.45)$ & $18.52(5.48)$ & .754 \\
\hline & 6 months & $19.43(4.54)$ & $18.63(6.21)$ & .69 \\
\hline & 12 months & $20.87(4.45)$ & $18.43(6.51)$ & .242 \\
\hline & 18 months & $21.33(2.74)$ & $19.75(5.61)$ & .336 \\
\hline
\end{tabular}

${ }^{*} P<.05$ statistically significant.

DTFC: dorzolamide/timolol fixed combination; LTFC: latanoprost/timolol fixed combination; IOP: intraocular pressure; POBF: pulsatile ocular blood flow.

damage has been reported in glaucoma patients [28-33]. In our study, we report differences in OPP and DPP between DTFC and LTFC; however, no significant differences were observed between LTFC and DTFC in terms of glaucoma progression during the 18-month followup.

Previous studies examining ocular blood flow and glaucoma progression reported structural abnormalities [34] preceding visual field damage. Hafez et al. [35] also concluded that rim perfusion might be reduced before manifestation of visual field defects. Several studies have shown abnormal retrobulbar vasculature in eyes with Glaucomatous Optic Neuropathy (GON) [36-40]. Satilmis et al. [41] showed that progression rate of glaucomatous visual field damage correlates with retrobulbar hemodynamic variables. Zeitz et al. [42] further showed that progressive glaucoma is associated with decreased blood flow velocities in the small retrobulbar vessels supplying the optic nerve head. We found increased blood flow velocities with combination treatment as compared to timolol baseline. DTFC arm had statistically significantly lower baseline OA-PSV and CRA-EDV as compared to LTFC baseline. After 1, 6, 12, and 18 months of combination treatment, the velocities in retrobulbar vessels increased as compared to baseline, but differences in velocities between two treatment arms were not statistically significant. In our study, SPCA-EDV was lower in progressing glaucoma patients as compared to stable glaucoma patients. We found statistically significant differences in RIs between the two treatment cohorts. DTFC showed statistically significant decrease in CRA and SPCA
RIs at 12- and 18-month visits as compared to LTFC. Nielsen and Nyborg [43] found that PG F2 $\alpha$ induces constriction in isolated bovine aqueous veins. Remky et al. [44] reported that reduction in retinal vessel diameters may account for an increase in retinal vascular resistance. An increase in vascular resistance might be related to vasoconstriction or vasospasm, vasosclerosis, reduction of the vessel diameters, or rheological factors leading to decreased volumetric flow. In our study, POBF that measures pulse volume was significantly higher in DTFC at 12 and 18-month visits compared to LTFC. Progressing glaucoma patients had 2.675 (SD 0.61) $\mu \mathrm{L}$ lower pulse volume when compared to stable glaucoma cases $(P=.0001)$. Our results indicate DTFC indeed increases markers of ocular blood flow and perfusion compared to LTFC but with no difference in possible markers of glaucoma progression during the followup period. Longer duration studies may be required to differentiate any possible (or lack thereof) ocular blood flow benefits.

The Beaver Dam study reported a positive correlation between systolic BP and IOP [45]. The Los Angeles Latino Eye Study [46] showed high systolic BP, low diastolic BP, and low OPP as risk factors for glaucoma progression. Data from EMGT [3] pointed to low systolic BP as a long-term predictor for glaucoma progression. Further, data from Thessaloniki Eye study [47] suggested BP status as an important independent factor initiating optic disc changes and/or as a contributing factor to glaucoma damage. In our study, we found no fluctuations or rise in IOP, but OPP and DPP at 1,6, and 18-month visits were statistically 
TABLE 4: Comparison of means between progressing and stable glaucoma patients at 18 months visit.

\begin{tabular}{|c|c|c|c|}
\hline Parameter at 18 month & $\begin{array}{c}\text { Mean in stable glaucoma patients } \\
\text { (St. deviation) }\end{array}$ & $\begin{array}{c}\text { Mean in progressing glaucoma patients } \\
\text { (St. deviation) }\end{array}$ & $\begin{array}{l}P \text { value } \\
(t \text {-test })\end{array}$ \\
\hline IOP & $15.32(2.46)$ & $16.73(3.04)$ & .171 \\
\hline $\mathrm{IOP} / \mathrm{POBF}$ & $14.73(3.5)$ & $16.88(3.89)$ & .123 \\
\hline $\mathrm{MD}(\mathrm{dB})$ & $-1.06(2.30)$ & $-2.01(2.13)$ & .257 \\
\hline $\operatorname{PSD}(\mathrm{dB})$ & $2.05(2.53)$ & $2.90(2.41)$ & .360 \\
\hline TSNIT $(\mu)$ & $53.59(5.28)$ & $50.96(7.10)$ & .254 \\
\hline NFI & $23.82(2.36)$ & $27.69(3.29)$ & $.0008^{*}$ \\
\hline SBP (mmHg) & $151.50(14.04)$ & $147.73(20.66)$ & .55 \\
\hline DBP (mmHg) & $88.44(6.42)$ & $83.53(8.23)$ & .077 \\
\hline OPP (mmHg) & $57.19(4.73)$ & $51.84(7.00)$ & $.019^{*}$ \\
\hline $\mathrm{DPP}(\mathrm{mmHg})$ & $73.06(6.57)$ & $64.85(8.82)$ & $.007^{*}$ \\
\hline OA_PSV & $32.26(3.15)$ & $29.82(3.28)$ & .048 \\
\hline OA_EDV & $9.19(4.98)$ & $7.25(2.01)$ & .197 \\
\hline OA_RI & $0.74(0.07)$ & $0.90(0.07)$ & $<.0001^{*}$ \\
\hline CRA_PSV & $19.96(7.36)$ & $15.61(6.28)$ & .099 \\
\hline CRA_EDV & $6.66(3.19)$ & $5.73(2.85)$ & .415 \\
\hline CRA_RI & $0.79(0.08)$ & $0.815(0.06)$ & .35 \\
\hline SPCA_PSV & $13.08(5.11)$ & $11.42(3.43)$ & .321 \\
\hline SPCA_EDV & $4.73(1.71)$ & $3.34(0.83)$ & $.011^{*}$ \\
\hline SPCA_RI & $0.77(0.20)$ & $0.76(0.20)$ & .893 \\
\hline AMPLITUDE & $4.62(1.48)$ & $4.62(1.44)$ & .985 \\
\hline PULSE VOLUME & $9.71(2.01)$ & $7.03(1.00)$ & $.0001^{*}$ \\
\hline POBF & $21.25(4.22)$ & $19.60(4.60)$ & .316 \\
\hline
\end{tabular}

DTFC: dorzolamide/timolol fixed combination; LTFC: latanoprost/timolol fixed combination; MD: mean deviation; PSD: pattern standard deviation; TSNIT: temporal, superior, nasal, inferior, temporal average; NFI: nerve fiber index. POBF: pulsatile ocular blood flow.

significantly higher in the DTFC group. The LTFC group had lower SBP at 1,6, and 18-month visits and diastolic BP at 1 and 6 month visits $(P<.05 ; t$-test $)$. Progressing patients had statistically significantly lower systolic BP, OPP, and DPP when compared to stable glaucoma cases. Calculating the magnitude of changes in OPP and DPP parameters compared to baseline values, we found them to be decreasing in $69.2 \%$ of progressing glaucoma cases. Our calculated sensitivity of decreased DPP was 0.7 and specificity 0.8 .

$\mathrm{BP}$ and ocular perfusion pressure tend to exhibit fluctuations during the day and night. Importantly, Choi et al. [48] reported that mean BP and OPP fluctuations were associated with reduced TSNIT and increased NFI. In our study, BP was measured at the same time of the day during all visits and statistically significant differences in $\mathrm{BP}$ and $\mathrm{OPP}$ parameters were seen at 1,6 , and 18 months but were not significant at 12 months between the two treatment groups. The LTFC group showed lower OPP and DPP and higher NFI as compared to DTFC at the 18 month visit (mean difference 7.80 (SD 3.69) $(P=.046)$. Accordingly, progressing glaucoma patients showed lower OPP and DPP and higher NFI (mean difference 8.87 (SD 3.94)); $P=.056$ ). Yet, despite differences in the nonpressure-related parameters, we found no difference in the percent of progression between the treatment groups. In addition, we also found a strong positive correlation between TSNIT average and BP and OPP parameters at 18-month visit. Interestingly, low OPP and DPP in progressing glaucoma patients had low sensitivity but rather high specificity. In our analysis, statistically significant Area Under ROC Curve (AUC) values were reported at 0.74 and 0.72 . While significant, these values should be further validated with a larger sample allowing for stratification into classified percentile ranges. The odds of higher NFI at the final 18 -month visit was nearly 14 times greater in patients with higher than 0.67 baseline CRA RI $(P=.028)$ and older age $(P=.021)$.

Current glaucoma medications are targeted to decrease the IOP and are not targeted to treat other hemodynamic parameters. In our study, we found some differences in structural outcomes between the two combination treatment regimes and according differences in BP, OPP, CRA, and SPCA RIs. Our study is a preliminary study and the data presented needs to be interpreted with caution. Increased resistance to flow in small retrobulbar vessels supplying the optic nerve is probably related to glaucoma progression, although this requires confirmation in larger longitudinal studies.

Possible limitations of the current study include the difficulty in defining glaucoma progression and specific limitations in each imaging technology used to assess ocular blood flow. We have matched markers of possible glaucoma progression, which may indicate but not actually represent 
glaucomatous progression. While the parameters may be associated with progression, they are not necessarily good in predicting progression. A risk factor must be strongly associated with a disorder to be a worthwhile screening test, and it is not unusual for a strong risk factor to fail to be a good screening tool. Larger group studies with longer followup, standardization of measurement techniques for glaucoma progression, and ocular blood flow parameters are required to elicit a clear understanding of vascular risk factors in glaucoma progression.

\section{Conflict of Interests}

The authors have no proprietary interest in any aspect of the products or devices mentioned herein.

\section{Acknowledgement}

This work is supported in part by an unrestricted grant from Research to Prevent Blindness. I. Janulevičiene and A. Harris have each previously received research grants from Merck \& Co, Inc., Whitehouse Station, NJ.

\section{References}

[1] S. Drange, D. R. Anderson, and M. Schulzer, "Risk factors for progression of visual field abnormalities in normal-tension glaucoma," American Journal of Ophthalmology, vol. 131, no. 6, pp. 699-708, 2001.

[2] A. Heijl, M. C. Leske, B. Bengtsson, L. Hyman, B. Bengtsson, and M. Hussein, "Reduction of intraocular pressure and glaucoma progression: results from the Early Manifest Glaucoma Trial," Archives of Ophthalmology, vol. 120, no. 10, pp. 12681279, 2002.

[3] M. C. Leske, A. Heijl, L. Hyman, B. Bengtsson, L. Dong, and Z. Yang, "Predictors of long-term progression in the early manifest glaucoma trial," Ophthalmology, vol. 114, no. 11, pp. 1965-1972, 2007.

[4] J. Flammer, "The vascular concept of glaucoma," Survey of Ophthalmology, vol. 38, no. 1, pp. S3-S6, 1994.

[5] S. S. Hayreh, "Progress in the understanding of the vascular etiology of glaucoma," Current Opinion in Ophthalmology, vol. 5, no. 2, pp. 26-35, 1994.

[6] European Glaucoma Society, Terminology and Guidelines for Glaucoma, Editrice DOGMA S.r.l., Savona, Italy, 3rd edition, 2008.

[7] N. N. Osborne, M. Ugarte, M. Chao et al., "Neuroprotection in relation to retinal ischemia and relevance to glaucoma," Survey of Ophthalmology, vol. 43, no. 6, pp. S102-S128, 1999.

[8] B. Siesky, A. Harris, N. Kheradiya, C. Rospigliosi, L. McCranor, and R. Ehrich, "The clinical significance of vascular factors in glaucoma," Journal of Current Glaucoma Practice, vol. 2, pp. 12-17, 2007.

[9] V. P. Costa, A. Harris, E. Stefánsson et al., "The effects of antiglaucoma and systemic medications on ocular blood flow," Progress in Retinal and Eye Research, vol. 22, no. 6, pp. 769-805, 2003.

[10] A. Harris, O. Arend, S. Arend, and B. Martin, "Effects of topical dorzolamide on retinal and retrobulbar hemodynamics," Acta Ophthalmologica Scandinavica, vol. 74, no. 6, pp. 569$572,1996$.
[11] A. Martinez, F. Gonzalez, C. Capeans, R. Perez, and M. Sanchez-Salorio, "Dorzolamide effect on ocular blood flow," Investigative Ophthalmology \& Visual Science, vol. 40, no. 6, pp. 1270-1275, 1999.

[12] A. Harris, O. Arend, L. Kagemann, M. Garrett, H. S. Chung, and B. Martin, "Dorzolamide, visual function and ocular hemodynamics in normal-tension glaucoma," Journal of Ocular Pharmacology and Therapeutics, vol. 15, no. 3, pp. 189-197, 1999.

[13] A. Harris, O. Arend, H. S. Chung, L. Kagemann, L. Cantor, and B. Martin, "A comparative study of betaxolol and dorzolamide effect on ocular circulation in normal-tension glaucoma patients," Ophthalmology, vol. 107, no. 3, pp. 430-434, 2000.

[14] A. Harris, C. P. Jonescu-Cuypers, L. Kagemann et al., "Effect of dorzolamide timolol combination versus timolol $0.5 \%$ on ocular bloodflow in patients with primary open-angle glaucoma," American Journal of Ophthalmology, vol. 132, no. 4, pp. 490-495, 2001.

[15] A. M. Avunduk, A. Sari, N. Akyol et al., "The one-month effects of topical betaxolol, dorzolamide and apraclonidine on ocular blood flow velocities in patients with newly diagnosed primary open-angle glaucoma," Ophthalmologica, vol. 215, no. 5, pp. 361-365, 2001.

[16] A. S. Bernd, L. E. Pillunat, A. G. Böhm, K. G. Schmidt, and G. Richard, "Okuläre hämodynamik und gesichtsfeld beim glaukom unter dorzolamid-therapie," Ophthalmologe, vol. 98, no. 5, pp. 451-455, 2001.

[17] L. E. Pillunat, A. G. Böhm, A. U. Köller, K. G. Schmidt, M. Klemm, and G. Richard, "Effect of topical dorzolamide on optic nerve head blood flow," Graefe's Archive for Clinical and Experimental Ophthalmology, vol. 237, no. 6, pp. 495-500, 1999.

[18] I. C. Bergstrand, A. Heijl, and A. Harris, "Dorzolamide and ocular blood flow in previously untreated glaucoma patients: a controlled double-masked study," Acta Ophthalmologica Scandinavica, vol. 80, no. 2, pp. 176-182, 2002.

[19] B. Siesky, A. Harris, E. Brizendine et al., "Literature review and meta-analysis of topical carbonic anhydrase inhibitors and ocular blood flow," Survey of Ophthalmology, vol. 54, no. 1, pp. 33-46, 2009.

[20] F. A. Medeiros, L. M. Alencar, L. M. Zangwill et al., "Detection of progressive retinal nerve fiber layer loss in glaucoma using scanning laser polarimetry with variable corneal compensation," Investigative Ophthalmology \& Visual Science, vol. 50, no. 4, pp. 1675-1681, 2009.

[21] L. M. Alencar, L. M. Zangwill, R. N. Weinreb et al., "A comparison of rates of change in neuroretinal rim area and retinal nerve fiber layer thickness in progressive glaucoma," Investigative Ophthalmology \& Visual Science, vol. 51, no. 7, pp. 3531-3539, 2010.

[22] I. Januleviciene, R. Ehrlich, B. Siesky, I. Nedzelskiené, and A. Harris, "Visual function, optic nerve structure, and ocular blood flow parameters after 1 year of glaucoma treatment ith fixed combinations," European Journal of Ophthalmology, vol. 19, no. 5, pp. 790-797, 2009.

[23] T. H. Williamson and A. Harris, "Color Doppler ultrasound imaging of the eye and orbit," Survey of Ophthalmology, vol. 40, no. 4, pp. 255-267, 1996.

[24] L. Pourcelot, "Applications of cliniques de l'examinen Doppler transcutane," INSERM, vol. 34, pp. 213-240, 1974.

[25] L. Pourcelot, "Indications of Doppler ultrasonography in the study of peripheral vessels," Revue du Praticien, vol. 25, no. 59, pp. 4671-4680, 1975. 
[26] T. A. Ciula, C. D. Regillo, and A. Harris, Retina and Optic Nerve Imaging, Lippincot Williams Wilkins, Philadelphia, Pa, USA, 2003.

[27] B. Siesky, A. Harris, D. Sines et al., "A comparative analysis of the effects of the fixed combination of timolol and dorzolamide versus latanoprost plus timolol on ocular hemodynamics and visual function in patients with primary open-angle glaucoma," Journal of Ocular Pharmacology and Therapeutics, vol. 22, no. 5, pp. 353-361, 2006.

[28] J. E. Grunwald, J. Piltz, S. M. Hariprasad, and J. DuPont, "Optic nerve and choroidal circulation in glaucoma," Investigative Ophthalmology \& Visual Science, vol. 39, no. 12, pp. 2329-2336, 1998.

[29] G. Michelson, M. J. Langhans, J. Harazny, and A. Dichtl, "Visual field defect and perfusion of the juxtapapillary retina and the neuroretinal rim area in primary open-angle glaucoma," Graefe's Archive for Clinical and Experimental Ophthalmology, vol. 236, no. 2, pp. 80-85, 1998.

[30] S. S. Hayreh, I. H. Revie, and J. Edwards, "Vasogenic origin of visual field defects and optic nerve changes in glaucoma," British Journal of Ophthalmology, vol. 54, no. 7, pp. 461-472, 1970.

[31] K. Yaoeda, M. Shirakashi, A. Fukushima et al., "Relationship between optic nerve head microcirculation and visual field loss in glaucoma," Acta Ophthalmologica Scandinavica, vol. 81, no. 3, pp. 253-259, 2003.

[32] M. T. Nicolela, S. M. Drance, S. J. A. Rankin, A. R. Buckley, and B. E. Walman, "Color Doppler imaging in patients with asymmetric glaucoma and unilateral visual field loss," American Journal of Ophthalmology, vol. 121, no. 5, pp. 502510, 1996.

[33] Y. Yamazaki and S. M. Drance, "The relationship between progression of visual field defects and retrobulbar circulation in patients with glaucoma," American Journal of Ophthalmology, vol. 124, no. 3, pp. 287-295, 1997.

[34] A. Sommer, J. Katz, H. A. Quigley et al., "Clinically detectable nerve fiber atrophy precedes the onset of glaucomatous field loss," Archives of Ophthalmology, vol. 109, no. 1, pp. 77-83, 1991.

[35] A. S. Hafez, R. L. G. Bizzarro, and M. R. Lesk, "Evaluation of optic nerve head and peripapillary retinal blood flow in glaucoma patients, ocular hypertensives, and normal subjects," American Journal of Ophthalmology, vol. 136, no. 6, pp. 10221031, 2003.

[36] Y. Yamazaki and F. Hayamizu, "Comparison of flow velocity of ophthalmic artery between primary open angle glaucoma and normal tension glaucoma," British Journal of Ophthalmology, vol. 79, no. 8, pp. 732-734, 1995.

[37] M. T. Nicolela, B. E. Walman, A. R. Buckley, and S. M. Drance, "Ocular hypertension and primary open-angle glaucoma: a comparative study of their retrobulbar blood flow velocity," Journal of Glaucoma, vol. 5, no. 5, pp. 308-310, 1996.

[38] S. J. A. Rankin, B. E. Walman, A. R. Buckley, and S. M. Drance, "Color Doppler imaging and spectral analysis of the optic nerve vasculature in glaucoma," American Journal of Ophthalmology, vol. 119, no. 6, pp. 685-693, 1995.

[39] P. Rojanapongpun, S. M. Drance, and B. J. Morrison, "Ophthalmic artery flow velocity in glaucomatous and normal subjects," British Journal of Ophthalmology, vol. 77, no. 1, pp. 25-29, 1993.

[40] H. J. Kaiser, A. Schoetzau, D. Stumpfig, and J. Flammer, "Blood-flow velocities of the extraocular vessels in patients with high-tension and normal-tension primary open-angle glaucoma," American Journal of Ophthalmology, vol. 123, no. 3, pp. 320-327, 1997.

[41] M. Satilmis, S. Orgül, B. Doubler, and J. Flammer, "Rate of progression of glaucoma correlates with retrobulbar circulation and intraocular pressure," American Journal of Ophthalmology, vol. 135, no. 5, pp. 664-669, 2003.

[42] O. Zeitz, P. Galambos, L. Wagenfeld et al., "Glaucoma progression is associated with decreased blood flow velocities in the short posterior ciliary artery," British Journal of Ophthalmology, vol. 90, no. 10, pp. 1245-1248, 2006.

[43] P. J. Nielsen and N. C. B. Nyborg, "Effects of prostaglandins in bovine isolated aqueous veins," Investigative Ophthalmology \& Visual Science, vol. 37, no. 3, p. S843, 1996.

[44] A. Remky, N. Plange, J. Klok, and O. Arend, "Retinal arterial diameters in patients with glaucoma," Spektrum der Augenheilkunde, vol. 18, no. 1, pp. 25-30, 2004.

[45] B. E. K. Klein, R. Klein, and M. D. Knudtson, "Intraocular pressure and systemic blood pressure: longitudinal perspective: the Beaver Dam Eye Study," British Journal of Ophthalmology, vol. 89, no. 3, pp. 284-287, 2005.

[46] V. Doshi, M. Ying-Lai, S. P. Azen, and R. Varma, "Sociodemographic, family history, and lifestyle risk factors for open-angle glaucoma and ocular hypertension. the Los Angeles Latino eye study," Ophthalmology, vol. 115, no. 4, pp. 639-647, 2008.

[47] F. Topouzis, A. L. Coleman, A. Harris et al., "Association of blood pressure status with the optic disk structure in nonglaucoma subjects: the Thessaloniki Eye Study," American Journal of Ophthalmology, vol. 142, no. 1, pp. 60-67, 2006.

[48] J. Choi, K. H. Kim, J. Jeong, H. S. Cho, C. H. Lee, and M. S. Kook, "Circadian fluctuation of mean ocular perfusion pressure is a consistent risk factor for normal-tension glaucoma," Investigative Ophthalmology \& Visual Science, vol. 48, no. 1, pp. 104-111, 2007. 


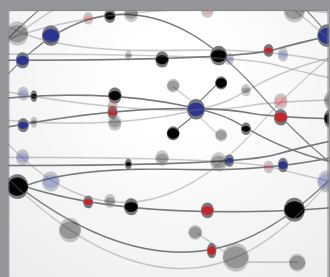

The Scientific World Journal
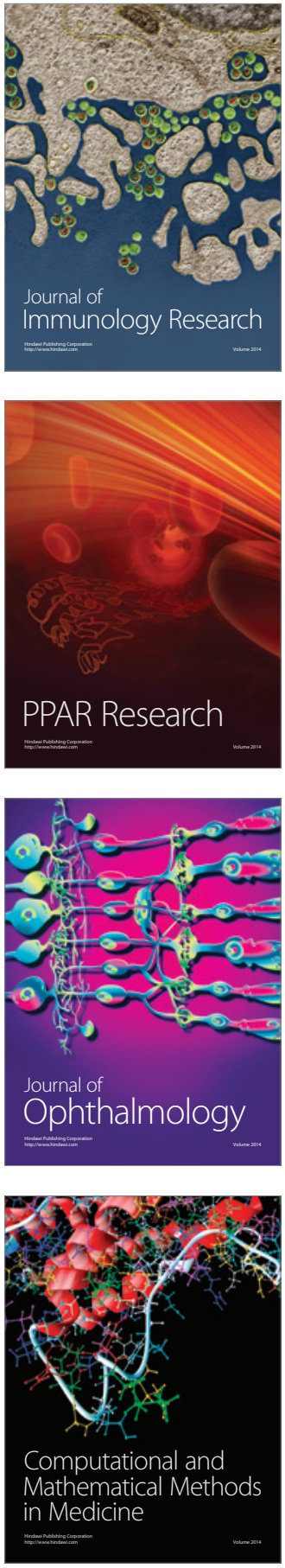

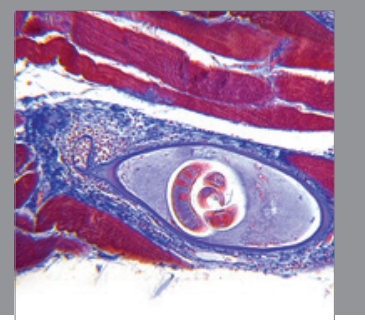

Gastroenterology

Research and Practice
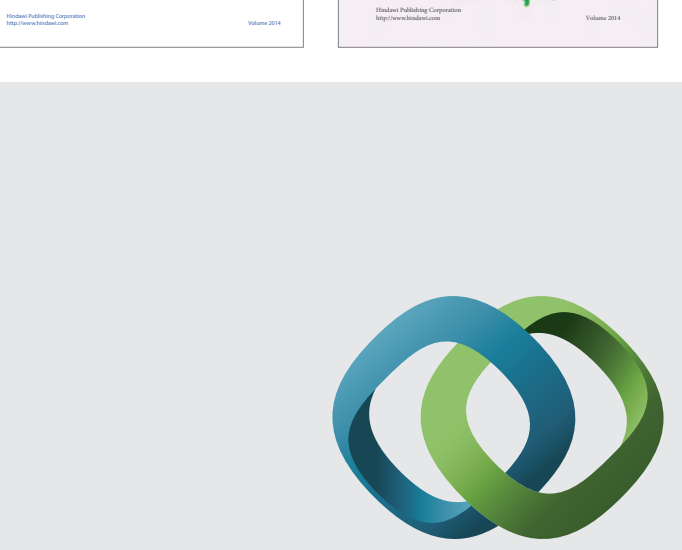

\section{Hindawi}

Submit your manuscripts at

http://www.hindawi.com
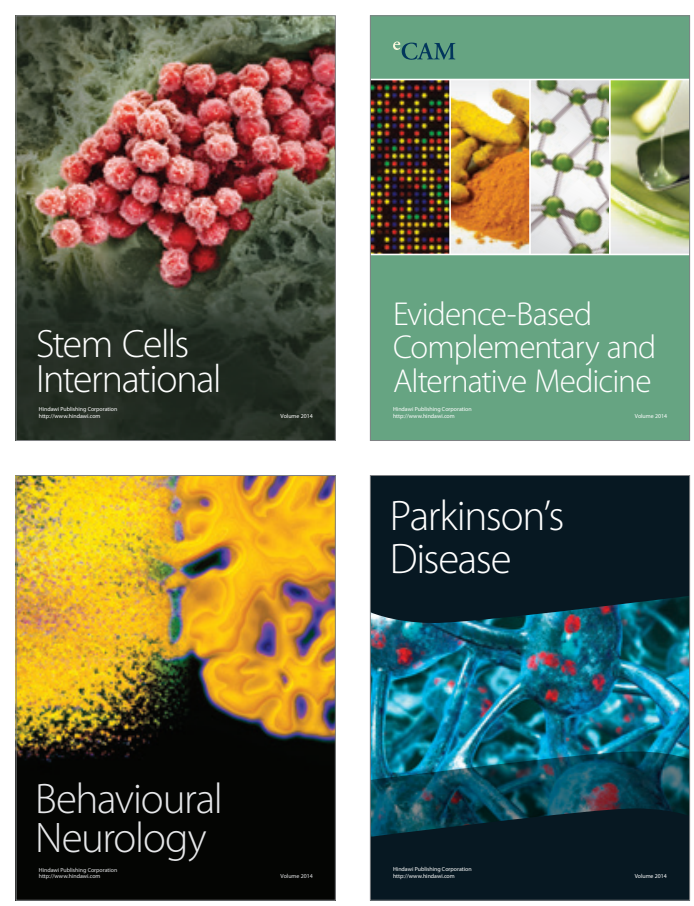

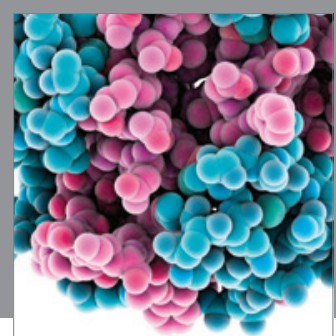

Journal of
Diabetes Research

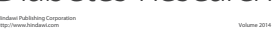

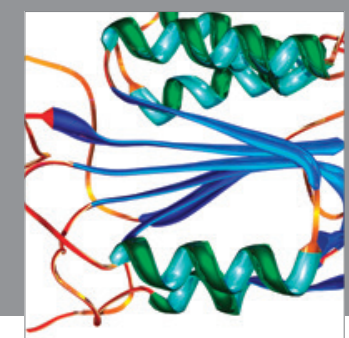

Disease Markers
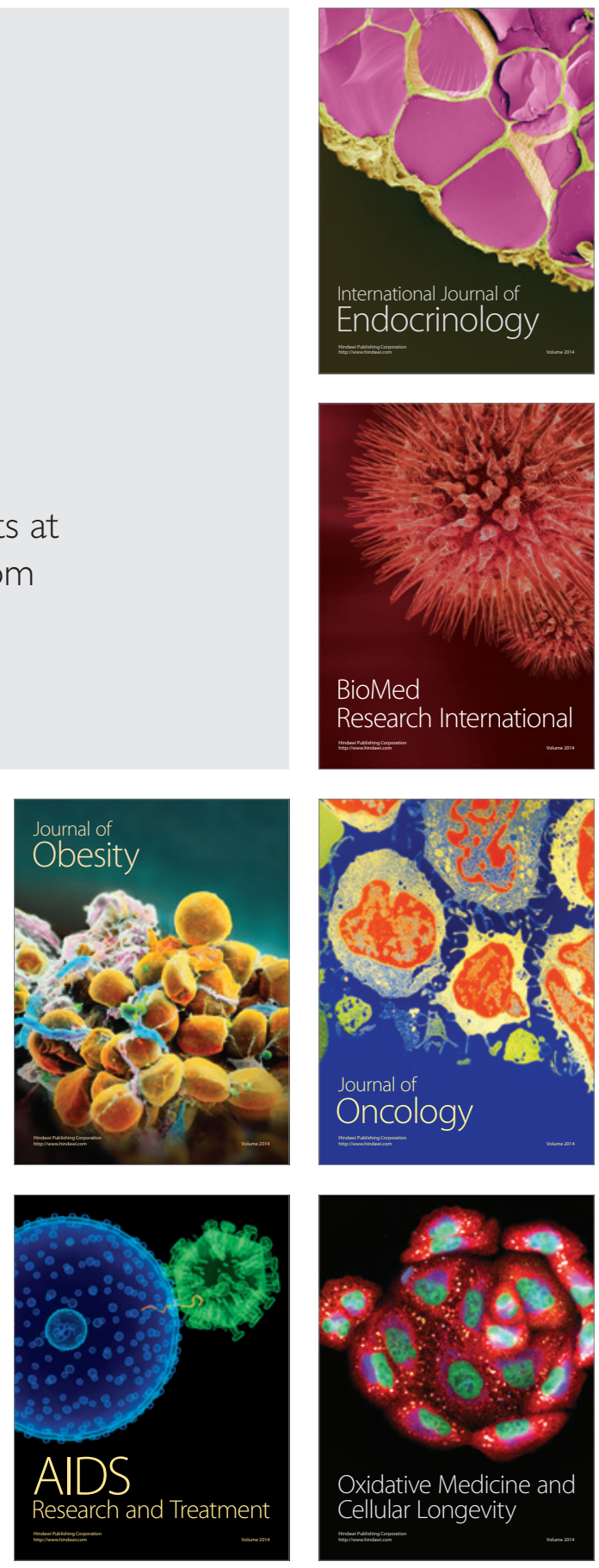